\title{
On Complex Gamma-Function Integrals
}

\author{
Sergey É. DERKACHOV ${ }^{\dagger}$ and Alexander N. MANASHOV ${ }^{\ddagger \dagger}$ \\ $\dagger$ St. Petersburg Department of Steklov Mathematical Institute of Russian Academy of Sciences, \\ Fontanka 27, 191023 St. Petersburg, Russia \\ E-mail: derkach@pdmi.ras.ru \\ ‡ Institut für Theoretische Physik, Universität Hamburg, D-22761 Hamburg, Germany \\ E-mail: alexander.manashov@desy.de
}

Received October 15, 2019, in final form January 14, 2020; Published online January 18, 2020

https://doi.org/10.3842/SIGMA.2020.003

\begin{abstract}
It was observed recently that relations between matrix elements of certain operators in the $\mathrm{SL}(2, \mathbb{R})$ spin chain models take the form of multidimensional integrals derived by R.A. Gustafson. The spin magnets with $\mathrm{SL}(2, \mathbb{C})$ symmetry group and $\mathrm{L}_{2}(\mathbb{C})$ as a local Hilbert space give rise to a new type of $\Gamma$-function integrals. In this work we present a direct calculation of two such integrals. We also analyse properties of these integrals and show that they comprise the star-triangle relations recently discussed in the literature. It is also shown that in the quasi-classical limit these integral identities are reduced to the duality relations for Dotsenko-Fateev integrals.
\end{abstract}

Key words: Mellin-Barnes integrals; star-triangle relation

2010 Mathematics Subject Classification: 33C70; 81R12

\section{Introduction}

The multidimensional integrals derived by R.A. Gustafson [27, 28] together with their $q$ - and elliptic analogues $[27,28,29,57,58,61]$ play an important role in different areas of physics and mathematics such as the theory of multi-variable orthogonal polynomials [62], Selberg type integrals and constant term identities [1,22], and supersymmetric dualities in quantum field theory [60]. Recently, a new field - noncompact spin magnets - was added to this list [15].

Models of this type appear in gauge field theories and have been under intense investigations in the last two decades, see references $[7,8]$. The mathematical description of such systems is welldeveloped and known as the quantum inverse scattering method(QISM) [20, 37, 38, 52, 53, 54]. Noncompact spin chains have an infinite-dimensional local Hilbert space and most conveniently can be analysed within the separation of variable (SoV) framework [53]. It was shown by Sklyanin that eigenfunctions of the entries of the monodromy matrix provide a suitable basis for solving the spectral problem for the spin chain Hamiltonian. Building such a basis for a generic spin chain is in itself a nontrivial problem and until recently the solution was available only for the spin chains with a rank-1 symmetry group. For the noncompact magnets of interest the corresponding eigenfunctions are known explicitly [9, 12, 14]. It should be mentioned here that recently there was a significant progress in constructing the SoV representation for compact chains of higher ranks, see [10, 24, 25, 34, 35, 40, 49].

In the $\mathrm{SL}(2, \mathbb{R})$ spin chin framework, Gustafson's integrals follow from identities for matrix elements of the shift operator [15]. Extension of this analysis to the $\mathrm{SL}(2, \mathbb{C})$ spin magnets leads to new integral identities $[16,17]$ which, up to the expected modification, are the exact replicas

This paper is a contribution to the Special Issue on Elliptic Integrable Systems, Special Functions and Quantum Field Theory. The full collection is available at https://www.emis.de/journals/SIGMA/elliptic-integrablesystems.html 
of the $\mathrm{SL}(2, \mathbb{R})$ integrals. The analysis, however, essentially depends on the completeness of the SoV representation. Proof of completeness is a rather complicated problem, see, e.g., [13, 36, 51]. Completeness for the closed $\mathrm{SL}(2, \mathbb{R})$ spin chain follows from that of the Toda chain [36] while for the $\mathrm{SL}(2, \mathbb{C})$ magnets of the length $N>2$ it is still an open question. A fruitful strategy seems to be to make use of Gustafson's integrals to prove completeness. To realize this one needs an independent derivation of the corresponding integrals. One of the purposes of this work is to provide such a derivation. We note also that complex gamma integrals were studied recently by V.F. Molchanov and Yu.A. Neretin [43] and Yu.A. Neretin [44, 45].

The paper is organized as follows: in Section 2, after setting the notations, we prove two integral identities which are direct analogs of Gustafson integrals associated with the classical $\operatorname{su}(N)$ and $\operatorname{sp}(N)$ Lie algebras [28]. We analyze the analytic properties of these integrals in Section 3 and derive two new integrals which are the $\mathrm{SL}(2, \mathbb{C})$ versions of Gustafson integrals [27] generalizing the second Barnes lemma. In Section 4 it will be shown that the $N=1,2$ integrals take, after some rewriting, the form of the star-triangle relations derived in $[5,32,33]$ as special limits of the elliptic star-triangle identity $[6,18,56,59]$. Section 5 is devoted to the study of the quasi-classical limit of the $\Gamma$-integrals. We show that in this limit the integrals are equivalent to a special case of the duality relation [2] for Dotsenko-Fateev (DF) integrals [19]. In Section 6 we present an elementary proof of the above duality relation and give some evidence which suggest that similar duality relations hold for the $\Gamma$-integrals. Section 7 is reserved for a summary.

\section{Gamma integrals}

\subsection{Definitions and basic properties}

Let $u, \bar{u}$ be a pair of complex numbers of the form

$$
u=\frac{n}{2}+\nu, \quad \bar{u}=-\frac{n}{2}+\nu,
$$

where $n$ is an integer and $\nu$ is complex number. We will use the notations $[u]=u-\bar{u}=n$ for the discrete part and $\langle u\rangle=\nu$ for the continuous part and put $\|u\|^{2}=-u \bar{u}=-\nu^{2}+n^{2} / 4$ so that for imaginary $\nu,\|u\|^{2} \geq 0$. The $\boldsymbol{\Gamma}$ function of the complex field $\mathbb{C}[23]$ is defined as

$$
\boldsymbol{\Gamma}(u, \bar{u})=\frac{\Gamma(u)}{\Gamma(1-\bar{u})}=\frac{\Gamma(n / 2+\nu)}{\Gamma(1+n / 2-\nu)}=(-1)^{n} \frac{\Gamma(-n / 2+\nu)}{\Gamma(1-n / 2-\nu)}=(-1)^{[u]} \Gamma(\bar{u}, u) .
$$

In what follows we will, for brevity, display only the first argument of the $\boldsymbol{\Gamma}$ function, i.e., $\boldsymbol{\Gamma}(u) \equiv \boldsymbol{\Gamma}(u, \bar{u})$. Hereafter the following functional relations will be useful

$$
\boldsymbol{\Gamma}(u) \boldsymbol{\Gamma}(1-u)=(-1)^{[u]}, \quad \boldsymbol{\Gamma}(u+1)=-u \bar{u} \boldsymbol{\Gamma}(u) .
$$

The $\boldsymbol{\Gamma}$ function appears in the generalization of Gustafson's integrals to the complex case.

The corresponding integrals take the following form $[16,17]$

$$
\begin{aligned}
\frac{1}{N !}{ }_{n_{1}, \ldots, n_{N} \in \mathbb{Z}+\frac{\sigma}{2}} \int_{-\mathrm{i} \infty}^{\mathrm{i} \infty} \frac{\prod_{m=1}^{N+1} \prod_{k=1}^{N} \boldsymbol{\Gamma}\left(z_{m}-u_{k}\right) \boldsymbol{\Gamma}\left(u_{k}+w_{m}\right)}{\prod_{m<j} \boldsymbol{\Gamma}\left(u_{m}-u_{j}\right) \boldsymbol{\Gamma}\left(u_{j}-u_{m}\right)} \frac{\mathrm{d} \nu_{1}}{2 \pi \mathrm{i}} \cdots \frac{\mathrm{d} \nu_{N}}{2 \pi \mathrm{i}} \\
=\frac{\prod_{k, j=1}^{N+1} \boldsymbol{\Gamma}\left(z_{k}+w_{j}\right)}{\boldsymbol{\Gamma}\left(\sum_{k=1}^{N+1}\left(z_{k}+w_{k}\right)\right)},
\end{aligned}
$$




$$
\begin{aligned}
\frac{1}{2^{N} N !} \sum_{n_{1}, \ldots, n_{N} \in \mathbb{Z}+\frac{\sigma}{2}} \int_{-\mathrm{i} \infty}^{\mathrm{i} \infty} \frac{\prod_{k=1}^{N} \prod_{m=1}^{2 N+2} \boldsymbol{\Gamma}\left(z_{m} \pm u_{k}\right)}{\prod_{k=1}^{N} \boldsymbol{\Gamma}\left( \pm 2 u_{k}\right) \prod_{k<j} \boldsymbol{\Gamma}\left( \pm u_{k} \pm u_{j}\right)} \frac{\mathrm{d} \nu_{1}}{2 \pi \mathrm{i}} \cdots \frac{\mathrm{d} \nu_{N}}{2 \pi \mathrm{i}} \\
=\frac{\prod_{j<k} \boldsymbol{\Gamma}\left(z_{j}+z_{k}\right)}{\boldsymbol{\Gamma}\left(\sum_{k=1}^{2 N+2} z_{k}\right)},
\end{aligned}
$$

where we put $\boldsymbol{\Gamma}(a \pm b) \equiv \boldsymbol{\Gamma}(a+b) \boldsymbol{\Gamma}(a-b), \boldsymbol{\Gamma}( \pm a \pm b) \equiv \boldsymbol{\Gamma}(a+b) \boldsymbol{\Gamma}(a-b) \Gamma(-a+b) \boldsymbol{\Gamma}(-a-b)$. We will refer to the integrals in the first and second lines as $I_{N}^{(1)}$ and $I_{N}^{(2)}$, respectively.

The variables $u_{k}, w_{m}, z_{m}$ have the form (2.1)

$$
u_{r}=\frac{n_{r}}{2}+\nu_{r}, \quad z_{j}=\frac{m_{j}}{2}+x_{j}, \quad w_{m}=\frac{\ell_{m}}{2}+y_{m}
$$

and similarly for the barred variables. However, $n_{r}, m_{i}, \ell_{m}$ are allowed take integer or half-integer values, simultaneously. Accordingly, the sums in (2.3) go over integers $(\sigma=0)$ or half-integers $(\sigma=1 / 2)$. For the first integral (2.3a) there is no difference between the integer/half-integer cases since they are related by the change of variables: $\left[u_{r}\right],\left[w_{m}\right],\left[z_{j}\right] \mapsto\left[u_{r}\right]+1 / 2,\left[w_{m}\right]-$ $1 / 2,\left[z_{j}\right]+1 / 2$ so that we will assume that the variables $\left[u_{k}\right],\left[w_{m}\right],\left[z_{m}\right]$ in the integral (2.3a) are integers.

The integration contours in (2.3) separate the series of " \pm " poles due to the $\boldsymbol{\Gamma}$ functions in the numerators. The poles are located at

$$
\nu_{r j}^{\mathrm{I},+}(p)=\frac{1}{2}\left|n_{r}-m_{j}\right|+x_{j}+p, \quad \nu_{r j}^{\mathrm{I},-}(p)=-\frac{1}{2}\left|n_{r}+\ell_{j}\right|-y_{j}-p, \quad p \geq 0,
$$

where $r \in\{1, N\}, j \in\{1, N+1\}$ for the first integral and

$$
\nu_{r j}^{\mathrm{II},+}(p)=\frac{1}{2}\left|n_{r}-m_{j}\right|+x_{j}+p, \quad \nu_{r j}^{\mathrm{II},-}(p)=-\frac{1}{2}\left|n_{r}+m_{j}\right|-x_{j}-p, \quad p \geq 0,
$$

where $r \in\{1, N\}, j \in\{1,2 N+2\}$, for the second one.

Let us discuss now the convergence properties of the integrals (2.3). Since the integrands are meromorphic functions and contours of integration avoid poles it is sufficient to analyse the region of large $u_{r}=n_{r} / 2+\mathrm{i} \nu_{r},\left\|u_{r}\right\|^{2}=\nu_{r}^{2}+n_{r}^{2} / 4 \rightarrow \infty$, only. With the help of (2.2) we simplify the denominators in the integrals (2.3) as follows

$$
\prod_{1 \leq i<k \leq N} \frac{1}{\boldsymbol{\Gamma}\left(u_{i}-u_{k}\right) \boldsymbol{\Gamma}\left(u_{k}-u_{i}\right)}=(-1)^{(N+1) \sum_{k}\left[u_{k}\right]} \prod_{1 \leq i<k \leq N}\left\|u_{i}-u_{k}\right\|^{2}
$$

and

$$
\begin{aligned}
& \prod_{k=1}^{N} \frac{1}{\boldsymbol{\Gamma}\left( \pm 2 u_{k}\right)} \prod_{1 \leq i<m \leq N} \frac{1}{\boldsymbol{\Gamma}\left( \pm u_{i} \pm u_{m}\right)} \\
& \quad=\varkappa_{N} 4^{N} \prod_{k=1}^{N}\left\|u_{k}\right\|^{2} \prod_{1 \leq i<m \leq N}\left\|u_{i}-u_{m}\right\|^{2}\left\|u_{i}+u_{m}\right\|^{2},
\end{aligned}
$$

where $\varkappa_{N}=1$ for the integer case and $\varkappa_{N}=(-1)^{N(N+1) / 2}$ for the half-integer case. Finally, taking into account that for large $u$

$$
\boldsymbol{\Gamma}(z-u) \boldsymbol{\Gamma}(u+w)=(-1)^{[z-u]} \frac{\boldsymbol{\Gamma}(u+w)}{\boldsymbol{\Gamma}(u-z)}
$$




$$
=(-1)^{[z-u]} u^{z+w-1}(-\bar{u})^{\bar{z}+\bar{w}-1}(1+O(1 /\|u\|))
$$

we conclude that the integrals (2.3) converge absolutely provided

$$
\sum_{j=1}^{N+1} \operatorname{Re}\left(x_{j}+y_{j}\right)<1 \quad \text { and } \quad \sum_{j=1}^{2 N+2} \operatorname{Re}\left(x_{j}\right)<1,
$$

respectively. From now on we assume that these conditions are satisfied.

\subsection{Determinant representation}

In this subsection we present the integrals (2.3) as determinants of 1-dimensional integrals. Such a representation will be useful in what follows. ${ }^{1}$ For its derivation let us denote by $\mathcal{Q}(u \mid z, w)$ the function

$$
\mathcal{Q}(u \mid z, w)=\prod_{k=1}^{N+1}(-1)^{[u]} \boldsymbol{\Gamma}\left(z_{k}-u\right) \boldsymbol{\Gamma}\left(u+w_{k}\right)
$$

and by $\mathcal{Q}_{i k}(z, w)$ its Mellin moments

$$
\mathcal{Q}_{i k}(z, w)=\int \mathcal{D} u u^{i-1}(-\bar{u})^{k-1} \mathcal{Q}(u \mid z, w), \quad i, k=1, \ldots, N .
$$

Here we introduced a short-hand notation for the integration measure

$$
\int \mathcal{D} u \equiv \sum_{n=-\infty}^{\infty} \int_{-\mathrm{i} \infty}^{\mathrm{i} \infty} \frac{\mathrm{d} \nu}{2 \pi \mathrm{i}} .
$$

Let $\mathcal{Q}_{N}(z, w)$ be the following $N \times N$ matrix constructed from the Mellin moments

$$
\mathcal{Q}_{N}(z, w)=\left(\begin{array}{ccc}
\mathcal{Q}_{11}(z, w) & \cdots & \mathcal{Q}_{1 N}(z, w) \\
\vdots & \ddots & \vdots \\
\mathcal{Q}_{N 1}(z, w) & \cdots & \mathcal{Q}_{N N}(z, w)
\end{array}\right)
$$

Rewriting the product on the r.h.s. of equation (2.5) as the product of two Vandermonde determinants

$$
\prod_{1 \leq i<k \leq N}\left\|u_{i}-u_{k}\right\|^{2}=\Delta(u) \Delta(-\bar{u}), \quad \Delta(u)=\operatorname{det}_{1 \leq i, j \leq N} u_{j}^{i-1}
$$

and taking into account the symmetry of the integrand in (2.3a) with respect to the permutations $u_{i} \leftrightarrow u_{j}$ we bring the first integral into the determinant form

$$
I_{N}^{(1)}=\int \mathcal{D} u_{1} \cdots \mathcal{D} u_{N} \Delta(-\bar{u}) \prod_{k=1}^{N} \mathcal{Q}\left(u_{k} \mid z, w\right) u_{k}^{k-1}=\operatorname{det} \mathcal{Q}_{N}(z, w) .
$$

Proceeding in a similar way one gets the determinant representation for the second integral as follows

$$
I_{N}^{(2)}=\varkappa_{N} \operatorname{det} \widetilde{\mathcal{Q}}_{N}(z)=\varkappa_{N} \operatorname{det}\left|\begin{array}{ccc}
\widetilde{\mathcal{Q}}_{11}(z) & \cdots & \widetilde{\mathcal{Q}}_{1 N}(z) \\
\vdots & \ddots & \vdots \\
\widetilde{\mathcal{Q}}_{N 1}(z) & \cdots & \widetilde{\mathcal{Q}}_{N N}(z)
\end{array}\right|,
$$

\footnotetext{
${ }^{1}$ The determinant representation for elliptic hypergeometric integrals was constructed in [47].
} 
where $\varkappa_{N}$ is a phase factor, see equation (2.6),

$$
\widetilde{\mathcal{Q}}_{i k}(z)=2 \int \mathcal{D} u u^{2 i-1}(-\bar{u})^{2 k-1} \widetilde{\mathcal{Q}}(u, z) \quad \text { and } \quad \widetilde{\mathcal{Q}}(u, z)=\prod_{j=1}^{2 N+2} \boldsymbol{\Gamma}\left(z_{j} \pm u\right) .
$$

Let us note here that the conditions (2.8) are equivalent to the requirement of absolute convergence of the Mellin moments $\mathcal{Q}_{N N}(z, w)$ and $\widetilde{\mathcal{Q}}_{N N}(z)$, respectively.

\subsection{Proof of identities (2.3)}

Calculating the integral (2.3a) we will assume that the parameters $z_{k}, w_{k}$ satisfy the conditions

$$
\sum_{k=1}^{N+1} \operatorname{Re}\left(z_{k}+w_{k}\right)<1 \quad \text { and } \quad \sum_{k=1}^{N+1} \operatorname{Re}\left(\bar{z}_{k}+\bar{w}_{k}\right)<1 .
$$

These conditions imply the condition (2.8), but do not follow from it and will be removed at the end of the calculation.

By virtue of (2.8) the integrals (2.9) are all absolutely convergent. We evaluate the integral over $\nu$ by closing the contour in the left half-plane and then computing the sum over the residues. Recalling that $u=n / 2+\nu$ and $\bar{u}=-n / 2+\nu$ one gets

$$
\begin{aligned}
M_{i k}(n)= & \int_{-\mathrm{i} \infty}^{\mathrm{i} \infty} u^{i-1}(-\bar{u})^{k-1} \mathcal{Q}(u \mid z, w) \frac{\mathrm{d} \nu}{2 \pi \mathrm{i}} \\
= & (-1)^{(N+1) n} \sum_{j=1}^{N+1} \sum_{p=0}^{\infty} \frac{(-1)^{p}}{p ! \bar{p}_{j} !}\left(-w_{j}-p\right)^{i-1}\left(\bar{w}_{j}+\bar{p}_{j}\right)^{k-1} \\
& \times \prod_{m=1}^{N+1} \frac{\Gamma\left(z_{m}+w_{j}+p\right)}{\Gamma\left(1-\bar{z}_{m}-\bar{w}_{j}-\bar{p}_{j}\right)} \prod_{m \neq j}^{N+1} \frac{\Gamma\left(w_{m}-w_{j}-p\right)}{\Gamma\left(1-\bar{w}_{m}+\bar{w}_{j}+\bar{p}_{j}\right)},
\end{aligned}
$$

where $\bar{p}_{j}=p+n+\ell_{j}$.

The first observation is that since the summand vanishes for $\bar{p}_{j}<0$, only the poles at $\nu_{r j}^{\mathrm{I},-}(p)$ given in (2.4), contribute to the integral and, second, that under the assumptions (2.12) the sum over $p$ converges uniformly on $n$. Therefore, to evaluate $\sum_{n} M_{i k}(n)$ we swap the summation over $n$ and $p$, change the summation variable from $n$ to $\bar{p}_{j}$ and finally suppress the index $j$ in $\bar{p}_{j}$ : $\bar{p}_{j} \mapsto \bar{p}$

$$
\sum_{n} M_{i k}(n)=\sum_{j=1}^{N+1} \sum_{p=0}^{\infty} \sum_{n=-\infty}^{\infty}(\ldots) \mapsto \sum_{j=1}^{N+1} \sum_{p=0}^{\infty} \sum_{\bar{p}_{j}=-\infty}^{\infty}(\ldots)=\sum_{j=1}^{N+1} \sum_{p=0}^{\infty} \sum_{\bar{p}=0}^{\infty}(\ldots) .
$$

The final answer can be written in the form

$$
\begin{aligned}
\mathcal{Q}_{i k}(z, w)= & \sum_{j=1}^{N+1}(-1)^{(N+1)\left[w_{j}\right]}\left(\prod_{m=1}^{N+1} \frac{\Gamma\left(z_{m}+w_{j}\right)}{\Gamma\left(1-\bar{z}_{m}-\bar{w}_{j}\right)}\right)\left(\prod_{\substack{m=1 \\
m \neq j}}^{N+1} \frac{\Gamma\left(w_{m}-w_{j}\right)}{\Gamma\left(1-\bar{w}_{m}+\bar{w}_{j}\right)}\right) \\
& \times\left(\sum_{p=0}^{\infty}\left(-w_{j}-p\right)^{i-1} \prod_{m=1}^{N+1} \frac{\left(z_{m}+w_{j}\right)_{p}}{\left(1-w_{m}+w_{j}\right)_{p}}\right) \\
& \times\left(\sum_{\bar{p}=0}^{\infty}\left(\bar{w}_{j}+\bar{p}\right)^{k-1} \prod_{m=1}^{N+1} \frac{\left(\bar{z}_{m}+\bar{w}_{j}\right)_{\bar{p}}}{\left(1-\bar{w}_{m}+\bar{w}_{j}\right)_{\bar{p}}}\right)
\end{aligned}
$$


where $(a)_{p}$ is the Pochhammer symbol. Thus $\mathcal{Q}_{i k}=\sum_{j=1}^{N+1} \mathcal{Q}_{i k}(j)=\sum_{j=1}^{N+1} \mathcal{Q}_{i}(j) \overline{\mathcal{Q}}_{k}(j)$. Hence the determinant can be represented as follows

$$
\begin{aligned}
\operatorname{det} \mathcal{Q} & =\sum_{j_{1}=1}^{N+1} \cdots \sum_{j_{N}=1}^{N+1} \operatorname{det}\left\|\mathcal{Q}_{i k}\left(j_{k}\right)\right\|=\sum_{\sigma \in S_{N+1}} \operatorname{det} \| \mathcal{Q}_{i k}\left(j_{\sigma(k)} \|\right. \\
& =\sum_{\sigma \in S_{N+1}}\left(\prod_{k=1}^{N} \overline{\mathcal{Q}}_{k}\left(j_{\sigma(k)}\right)\right) \operatorname{det}\left\|\mathcal{Q}_{i}\left(j_{\sigma(k)}\right)\right\|,
\end{aligned}
$$

where we take into account that $\operatorname{det}\left\|Q_{i k}\left(j_{k}\right)\right\|=0$ whenever $j_{m}=j_{n}$ for $n \neq m$. Then making use of (2.13) one can bring (2.11) into the following form

$$
\begin{aligned}
I_{N}^{(1)}= & \frac{1}{N !} \sum_{\sigma \in S_{N+1}}(-1){ }^{(N+1) \sum_{s=1}^{N}\left[w_{\sigma(s)}\right]} \prod_{k=1}^{N}\left(\prod_{j=1}^{N+1} \frac{\Gamma\left(z_{j}+w_{\sigma(k)}\right)}{\Gamma\left(1-\bar{z}_{j}-\bar{w}_{\sigma(k)}\right)} \prod_{\substack{m=1 \\
m \neq \sigma(k)}}^{N+1} \frac{\Gamma\left(w_{m}-w_{\sigma(k)}\right)}{\Gamma\left(1-\bar{w}_{m}+\bar{w}_{\sigma(k)}\right)}\right) \\
& \times \sum_{p_{1}, \ldots, p_{N}=0}^{\infty}\left(\prod_{k<m}\left(w_{\sigma(k)}+p_{k}-w_{\sigma(m)}-p_{m}\right)\right) \prod_{m=1}^{N+1} \prod_{k=1}^{N} \frac{\left(z_{m}+w_{\sigma(k)}\right)_{p_{k}}}{\left(1-w_{m}+w_{\sigma(k)}\right)_{p_{k}}} \\
& \times \sum_{\bar{p}_{1}, \ldots, \bar{p}_{N}=0}^{\infty}\left(\prod_{k<m}\left(\bar{w}_{\sigma(m)}+\bar{p}_{m}-\bar{w}_{\sigma(k)}-\bar{p}_{k}\right)\right) \prod_{m=1}^{N+1} \prod_{k=1}^{N} \frac{\left(\bar{z}_{m}+\bar{w}_{\sigma(k)}\right)_{\bar{p}_{k}}}{\left(1-\bar{w}_{m}+\bar{w}_{\sigma(k)}\right)_{\bar{p}_{k}}} .
\end{aligned}
$$

The infinite sums over $\{p\},\{\bar{p}\}$ can be evaluated by Milne's U(n) Gauss summation [42], see also [28, equation (5.8)] and [50],

$$
\begin{aligned}
\sum_{p_{1}, \ldots, p_{N}=0}^{\infty}\left(\prod_{1 \leq k<m \leq N}\left(\alpha_{\sigma(k)}+p_{k}-\alpha_{\sigma(m)}-p_{m}\right)\right) \prod_{i=1}^{N+1} \prod_{k=1}^{N} \frac{\left(\beta_{i}+\alpha_{\sigma(k)}\right)_{p_{k}}}{\left(1-\alpha_{i}+\alpha_{\sigma(k)}\right)_{p_{k}}} & \Gamma\left(1-\sum_{k=1}^{N+1}\left(\alpha_{k}+\beta_{k}\right)\right) \prod_{i=1}^{N} \Gamma\left(1+\alpha_{\sigma(i)}-\alpha_{\sigma(N+1)}\right) \\
= & \frac{\left.\Gamma+\beta_{i}-\alpha_{\sigma(N+1)}\right)}{\prod_{i=1}^{N+1} \Gamma\left(1-\beta_{i}\right.} .
\end{aligned}
$$

Using (6.5) we obtain the following representation for the integral $I_{N}^{(1)}$

$$
\begin{aligned}
I_{N}^{(1)}= & \frac{1}{N !} \sum_{\sigma \in S_{N+1}}(-1)^{(N+1) \sum_{s=1}^{N}\left[w_{\sigma(s)}\right]} \prod_{k=1}^{N}\left(\prod_{i=1}^{N+1} \frac{\Gamma\left(z_{i}+w_{\sigma(k)}\right)}{\Gamma\left(1-\bar{z}_{i}-\bar{w}_{\sigma(k)}\right)} \prod_{\substack{m=1 \\
m \neq \sigma(k)}}^{N+1} \frac{\Gamma\left(w_{m}-w_{\sigma(k)}\right)}{\Gamma\left(1-\bar{w}_{m}+\bar{w}_{\sigma(k)}\right)}\right) \\
& \times \prod_{1 \leq m<j \leq N}\left(w_{\sigma(m)}-w_{\sigma(j)}\right) \Gamma\left(1-\sum_{k=1}^{N+1}\left(z_{k}+w_{k}\right)\right) \frac{\prod_{k=1}^{N} \Gamma\left(1+w_{\sigma(k)}-w_{\sigma(N+1)}\right)}{\left.\prod_{k=1}^{N+1} \Gamma\left(1-z_{k}-w_{\sigma(N+1)}\right)\right)} \\
& \times \prod_{1 \leq m<j \leq N}\left(\bar{w}_{\sigma(j)}-\bar{w}_{\sigma(m)}\right) \Gamma\left(1-\sum_{k=1}^{N+1}\left(\bar{z}_{k}+\bar{w}_{k}\right)\right) \frac{\prod_{k=1}^{N} \Gamma\left(1+\bar{w}_{\sigma(k)}-\bar{w}_{\sigma(N+1)}\right)}{\prod_{k=1}^{N+1} \Gamma\left(1-\bar{z}_{k}-\bar{w}_{\sigma(N+1)}\right)} .
\end{aligned}
$$


After some simplifications this can be written as

$$
I_{N}^{(1)}=\frac{\Gamma\left(1-\sum_{k=1}^{N+1}\left(\bar{z}_{k}+\bar{w}_{k}\right)\right)}{\Gamma\left(\sum_{k=1}^{N+1}\left(z_{k}+w_{k}\right)\right)} \prod_{i, k=1}^{N+1} \frac{\Gamma\left(z_{i}+w_{k}\right)}{\Gamma\left(1-\bar{z}_{i}-\bar{w}_{k}\right)} \frac{R_{N}}{N ! \sin \pi\left(\sum_{k=1}^{N+1}\left(z_{k}+w_{k}\right)\right)},
$$

where

$$
\begin{aligned}
R_{N}= & \sum_{\sigma \in S_{N+1}} \frac{\prod_{k=1}^{N+1} \sin \pi\left(z_{\sigma(k)}+w_{\sigma(N+1)}\right)}{\prod_{k=1}^{N} \sin \pi\left(w_{\sigma(N+1)}-w_{\sigma(k)}\right)} \\
& \times(-1)^{(N+1) \sum_{s=1}^{N}\left[w_{\sigma(s)}\right]} \prod_{1 \leq k<j \leq N} \frac{\sin \pi\left(\bar{w}_{\sigma(j)}-\bar{w}_{\sigma(k)}\right)}{\sin \pi\left(w_{\sigma(j)}-w_{\sigma(k)}\right)} .
\end{aligned}
$$

Taking into account that $w_{k}-\bar{w}_{k}$ is an integer one finds that the last product in (2.15) yields $(-1)^{(N-1)} \sum_{s=1}^{N}\left[w_{\sigma(s)}\right]$ which cancels the second factor in $(2.15)$. In the last step we use the [28, Lemma 5.10] which states that

$$
\sum_{\sigma \in S_{N+1}} \frac{\prod_{k=1}^{N+1} \sin \pi\left(\beta_{k}+\alpha_{\sigma(N+1)}\right)}{\prod_{k=1}^{N} \sin \pi\left(\alpha_{\sigma(N+1)}-\alpha_{\sigma(k)}\right)}=N ! \sin \pi \sum_{k=1}^{N+1}\left(\alpha_{k}+\beta_{k}\right) .
$$

It results in

$$
R_{N}=N ! \sin \pi \sum_{k=1}^{N+1}\left(z_{k}+w_{k}\right)
$$

so that we get the required result for $I_{N}^{(1)}$

$$
I_{N}^{(1)}=\frac{\prod_{k, j=1}^{N+1} \boldsymbol{\Gamma}\left(z_{k}+w_{j}\right)}{\boldsymbol{\Gamma}\left(\sum_{k=1}^{N+1}\left(z_{k}+w_{k}\right)\right)} .
$$

Finally by analytic continuation in the $\nu_{k}$ the assumptions (2.12) can be relaxed to the condition (2.8).

One sees that the crucial point in the proof of $(2.3 \mathrm{a})$ is the factorization of the double sum arising after evaluating the integral over $\nu$ by residue theorem into the product of two infinite sums, see equation (2.13). We remark that this factorization was first noticed by Ismagilov [30,31], see also [45]. After this property is established the further analysis follows along the lines of [28].

The proof of the identity $(2.3 \mathrm{~b})$ proceeds along the same lines so we will not go into details and only highlight the essential differences. First, we assume that the parameters $z_{k}$ satisfy the conditions

$$
\sum_{k=1}^{2 N+2} \operatorname{Re}\left(z_{k}\right)<1 \quad \text { and } \quad \sum_{k=1}^{2 N+2} \operatorname{Re}\left(\bar{z}_{k}\right)<1 .
$$


After evaluating the integrals over $\nu$ using residue calculus, and carrying out some minor simplifications, we obtain

$$
\begin{aligned}
I_{N}^{(2)}= & \frac{2^{N} \varkappa_{N}}{N !}(-1)^{N} \sum_{\pi}\left(\left\{\sum_{y_{1}, \ldots, y_{N}=0}^{\infty}\left(\prod_{j=1}^{N}\left(z_{\pi(j)}+y_{j}\right) \prod_{1 \leq i<j \leq N}\left(z_{\pi(i)}+y_{i} \pm\left(z_{\pi(j)}+y_{j}\right)\right)\right)\right.\right. \\
& \left.\times \prod_{k=1}^{N} \frac{(-1)^{y_{k}}}{y_{k} !} \Gamma\left(2 z_{\pi(k)}+y_{k}\right) \prod_{\substack{j=1 \\
j \neq \pi(k)}}^{2 N+2} \Gamma\left(z_{j} \pm\left(z_{\pi(k)}+y_{k}\right)\right)\right\} \\
& \times\left\{\sum_{\bar{y}_{1}, \ldots, \bar{y}_{N}=0}^{\infty}\left(\prod_{j=1}^{N}\left(\bar{z}_{\pi(j)}+\bar{y}_{j}\right) \prod_{1 \leq i<j \leq N}\left(\bar{z}_{\pi(i)}+\bar{y}_{i} \pm\left(\bar{z}_{\pi(j)}+\bar{y}_{j}\right)\right)\right)\right. \\
& \left.\left.\times \prod_{k=1}^{N} \frac{1}{\bar{y}_{k} !} \frac{1}{\Gamma\left(1-2 \bar{z}_{\pi(k)}-\bar{y}_{k}\right)} \prod_{\substack{j=1 \\
j \neq \pi(k)}}^{2 N+2} \frac{1}{\Gamma\left(1-\bar{z}_{j} \pm\left(\bar{z}_{\pi(k)}+\bar{y}_{k}\right)\right)}\right\}\right),
\end{aligned}
$$

where $\pi$ is an injective map from $\{1, \ldots, N\}$ to $\{1, \ldots, 2 N+2\}$. The sums over $y_{j}, \bar{y}_{j}$ can be evaluated with the help of the following hypergeometric series summation formula, see [26, 28]

$$
\begin{gathered}
\sum_{y_{1}, \ldots, y_{n}=-\infty}^{\infty}\left\{\prod_{j=1}^{n} \frac{z_{j}+y_{j}}{z_{j}} \prod_{1 \leq i<j \leq n} \frac{z_{i}+y_{i} \pm\left(z_{j}+y_{j}\right)}{z_{i} \pm z_{j}} \prod_{i=1}^{2 n+2} \prod_{j=1}^{n} \frac{\Gamma\left(w_{i} \pm\left(z_{j}+y_{j}\right)\right)}{\Gamma\left(w_{i} \pm z_{j}\right)}\right\} \\
=\frac{\Gamma\left(1-\sum_{k=1}^{2 n+2} w_{i}\right) \prod_{i=1}^{2 n+2} \prod_{j=1}^{n} \Gamma\left(1-w_{i} \pm z_{j}\right)}{\prod_{j=1}^{N} \Gamma\left(1 \pm 2 z_{j}\right) \prod_{1 \leq i<j \leq n} \Gamma\left(1 \pm z_{i} \pm z_{j}\right) \prod_{1 \leq i<j \leq 2 n+2} \Gamma\left(1-w_{i}-w_{j}\right)} .
\end{gathered}
$$

Collecting all factors we obtain

$$
I_{N}^{(2)}=\frac{\prod_{1 \leq i<j \leq 2 N+2} \Gamma\left(z_{i}+z_{j}\right)}{\Gamma\left(1-\sum_{k} z_{k}\right)} \times T_{N},
$$

where

$$
T_{N}=\frac{(-1)^{N}}{2^{N} N !} \frac{\prod_{1 \leq i<j \leq 2 N+2} \sin \pi\left(z_{i}+z_{j}\right)}{\sin \pi\left(\sum_{k} z_{k}\right)} \sum_{\pi} \frac{\prod_{1 \leq i<j \leq N} \sin ^{2} \pi\left(z_{\pi(i)} \pm z_{\pi(j)}\right) \prod_{j=1}^{N} \sin \pi\left(2 z_{\pi(j)}\right)}{\prod_{j=1}^{N} \prod_{\substack{i=1 \\ i \neq \pi(j)}}^{2 N+2} \sin \pi\left(z_{i} \pm z_{\pi(j)}\right)} .
$$

One can show that $T_{N}=1$ by taking the limit $q \rightarrow 1$ of the identities in [28, equations (7.11) and (7.12)]. Finally, the conditions $(2.16)$ can be relaxed to (2.8) by analytic continuation in the $\nu_{k}$.

\section{Limiting cases}

In this section we derive two more integrals which are replicas of the integrals [27, equations (3.2) and (5.4)]. We show that in the complex case these integrals are intrinsically related to the integrals (2.3). This property is not seen in the $\mathrm{SL}(2, \mathbb{R})$ setup. 
We start our analysis with the integral (2.3a) and introduce the variables, complex $\zeta$ and integer $\eta$, as follows

$$
\zeta+\frac{\eta}{2}=\sum_{k=1}^{N+1}\left(z_{k}+w_{k}\right), \quad \zeta-\frac{\eta}{2}=\sum_{k=1}^{N+1}\left(\bar{z}_{k}+\bar{w}_{k}\right) .
$$

The r.h.s. of (2.3a) is a meromorphic function of $\zeta$ with poles located at the points $\zeta_{p}=$ $|1+\eta / 2|+p, p \in \mathbb{N}_{+}$. For $\eta=0$ and $\zeta$ close to 1 the r.h.s. of (2.3a) takes the form

$$
I_{N}^{(1)}=\frac{1}{1-\zeta} \prod_{k, j=1}^{N+1} \boldsymbol{\Gamma}\left(z_{k}+w_{j}\right)+\cdots,
$$

where $z_{k}, w_{k}$ obey the constraint $\sum_{k=1}^{N+1}\left(z_{k}+w_{k}\right)=\sum_{k=1}^{N+1}\left(\bar{z}_{k}+\bar{w}_{k}\right)=1$. At the same time, only the element $\mathcal{Q}_{N N}$ of the matrix (2.10) becomes singular at this point. The corresponding integral diverges at large $\nu$ and $n$ as $\zeta \rightarrow 1$. Indeed, taking into account equation (2.7) one finds

$$
\mathcal{Q}(u \mid z, w)=(-1)^{\sum_{k}\left[z_{k}\right]} u^{\zeta-N-1}(-\bar{u})^{\zeta-N-1}(1+O(1 /\|u\|)) .
$$

Thus for $\zeta \rightarrow 1$

$$
\mathcal{Q}_{N, N}(z, w) \simeq(-1)^{\sum_{k}\left[z_{k}\right]} \frac{1}{\pi} \int_{r>\Lambda} \frac{1}{r^{4-2 \zeta}} \mathrm{d} x \mathrm{~d} y+\cdots=\frac{1}{1-\zeta}(-1)^{\sum_{k}\left[z_{k}\right]}+\cdots
$$

and therefore

$$
I_{N}^{(1)} \underset{\zeta \rightarrow 1}{=} \mathcal{Q}_{N N}(z, w) \times \operatorname{det} \widehat{\mathcal{Q}}_{N-1}(z, w)+\text { finite terms }
$$

Here $\widehat{\mathcal{Q}}_{N-1}$ is the main $N-1$ minor of the matrix $\mathcal{Q}_{N}$. Comparing the residues at $\zeta=1$ in equation (3.3) and (3.2) we obtain the following identity

$$
\operatorname{det} \widehat{\mathcal{Q}}_{N-1}(z, w)=(-1)^{\sum_{k}\left[z_{k}\right]} \prod_{k, j=1}^{N+1} \boldsymbol{\Gamma}\left(z_{k}+w_{j}\right),
$$

which holds provided $\sum_{k=1}^{N+1}\left(z_{k}+w_{k}\right)=\sum_{k=1}^{N+1}\left(\bar{z}_{k}+\bar{w}_{k}\right)=1$. Rewriting the 1.h.s. of $(3.4)$ in an integral form one obtains

$$
\begin{aligned}
& \frac{1}{(N-1) !} \int \mathcal{D} u_{1} \cdots \mathcal{D} u_{N-1} \prod_{p=1}^{N-1}(-1)^{n_{p}} \frac{\prod_{j=1}^{N+1} \prod_{k=1}^{N-1} \boldsymbol{\Gamma}\left(z_{j}-u_{k}\right) \boldsymbol{\Gamma}\left(u_{k}+w_{j}\right)}{\prod_{k<j} \boldsymbol{\Gamma}\left(u_{k}-u_{j}\right) \boldsymbol{\Gamma}\left(u_{j}-u_{k}\right)} \\
& \quad=(-1)^{\sum_{m}\left[z_{m}\right]} \prod_{k, j=1}^{N+1} \boldsymbol{\Gamma}\left(z_{k}+w_{j}\right) .
\end{aligned}
$$

This integral is an analog of the integral [27, equation (3.2)]. Indeed, replacing the variable $z_{N+1}$ by $z_{N+1}=1-\sum_{k=1}^{N} z_{k}-\sum_{m=1}^{N+1} w_{m}$ and using the relation (2.2) one can bring equation (3.5) into the form which is a replica of [27, equation (3.2)]

$$
\frac{1}{(N-1) !} \int \mathcal{D} u_{1} \cdots \mathcal{D} u_{N-1} \frac{\prod_{k=1}^{N-1} \prod_{m=1}^{N} \prod_{j=1}^{N+1} \boldsymbol{\Gamma}\left(z_{m}-u_{k}\right) \boldsymbol{\Gamma}\left(u_{k}+w_{j}\right)}{\prod_{m=1}^{N-1} \boldsymbol{\Gamma}\left(\gamma+u_{m}\right) \prod_{k<j} \boldsymbol{\Gamma}\left(u_{k}-u_{j}\right) \boldsymbol{\Gamma}\left(u_{j}-u_{k}\right)}
$$




$$
=\frac{\prod_{k=1}^{N} \prod_{j=1}^{N+1} \boldsymbol{\Gamma}\left(z_{k}+w_{j}\right)}{\prod_{j=1}^{N+1} \boldsymbol{\Gamma}\left(\gamma-w_{j}\right)}
$$

where $\gamma=\sum_{k=1}^{N} z_{k}+\sum_{m=1}^{N+1} w_{m}$

The analysis of the second integral, equation $(2.3 \mathrm{~b})$, proceeds along the same lines so we give only a brief account. Similar to (3.1) we define variables, $\eta$ and $\zeta$, by $\zeta+\eta / 2=\sum_{k=1}^{2 N+2} z_{k}$. The l.h.s. and r.h.s. of equation $(2.3 \mathrm{~b})$ have a pole at $\zeta=1$ (for $\eta=0$ ). Comparing the corresponding residues we get

$$
\begin{aligned}
& \frac{1}{2^{N-1}(N-1) !} \int_{ \pm} \mathcal{D} u_{1} \cdots \mathcal{D} u_{N-1} \frac{\prod_{k=1}^{N-1} \prod_{j=1}^{2 N+2} \Gamma\left(z_{j} \pm u_{k}\right)}{\prod_{k=1}^{N-1} \Gamma\left( \pm 2 u_{k}\right)} \prod_{j<k} \boldsymbol{\Gamma}\left( \pm u_{k} \pm u_{j}\right) \\
& \quad= \pm \prod_{1 \leq j<k \leq 2 N+2} \Gamma\left(z_{j}+z_{k}\right),
\end{aligned}
$$

where $\sum_{k=1}^{2 N+2} z_{k}=\sum_{k=1}^{2 N+2} \bar{z}_{k}=1$ and the subscript \pm at the integral sign indicates that the sum goes over either integer $n$ (plus) or half-integer $n$ (minus). Introducing the variable $\gamma=\sum_{k=1}^{2 N+1} z_{k}$, one can rewrite this integral in the form identical to the integral [27, equation (5.4)]

$$
\begin{gathered}
\frac{2^{1-N}}{(N-1) !} \int_{ \pm} \mathcal{D} u_{1} \cdots \mathcal{D} u_{N-1} \frac{\prod_{k=1}^{N-1} \prod_{j=1}^{2 N+1} \boldsymbol{\Gamma}\left(z_{j} \pm u_{k}\right)}{\prod_{k=1}^{N-1} \boldsymbol{\Gamma}\left(\gamma \pm u_{k}\right) \boldsymbol{\Gamma}\left( \pm 2 u_{k}\right) \prod_{j<k} \boldsymbol{\Gamma}\left( \pm u_{k} \pm u_{j}\right)} \\
=\frac{\prod_{1 \leq j<k \leq 2 N+1} \boldsymbol{\Gamma}\left(z_{j}+z_{k}\right)}{\prod_{k=1}^{N+3} \boldsymbol{\Gamma}\left(\gamma-z_{k}\right)} .
\end{gathered}
$$

Thus in the $\mathrm{SL}(2, \mathbb{C})$ setup the integrals (3.5), (3.6) and (3.7), (3.8) are intrinsically related to the integrals (2.3). For $N=2$ the relation (3.8) was derived by Sarkissian and Spiridonov [55].

\section{Star-triangle relation}

In this section we show that the star-triangle relations with the Boltzmann weights given by a product of $\Gamma$-functions $[5,32,33]$ follow in a rather straightforward way from the integrals $(2.3)$. The star-triangle relation underlies exact solvability of various two-dimensional lattice models, see references [3,4] for a review. Here we recall such a relation inherent to the noncompact $\mathrm{SL}(2, \mathbb{C})$ spin chain magnets $[12]$.

Let $s_{\alpha}(z) \equiv s_{\alpha, \bar{\alpha}}(z, \bar{z})$ be a function of the complex variables $z=x+\mathrm{i} y, \bar{z} \equiv z^{*}=x-\mathrm{i} y$,

$$
\boldsymbol{s}_{\alpha}(z)=[z]^{-\alpha} \equiv z^{-\alpha} \bar{z}^{-\bar{\alpha}}, \quad[\alpha]=\alpha-\bar{\alpha} \in \mathbb{Z} .
$$


The function $s_{\alpha}(z)$ is a single valued function on the complex plane and in the physics literature it is usually called a propagator. ${ }^{2}$ It satisfies two identities:

- the chain relation

$$
\frac{1}{\pi} \int \boldsymbol{s}_{\alpha_{1}}\left(z_{1}-z\right) \boldsymbol{s}_{\alpha_{2}}\left(z-z_{2}\right) \mathrm{d}^{2} z=\frac{\boldsymbol{\Gamma}\left(1-\alpha_{1}\right) \boldsymbol{\Gamma}\left(1-\alpha_{2}\right)}{\boldsymbol{\Gamma}\left(2-\alpha_{1}-\alpha_{2}\right)} \boldsymbol{s}_{\alpha_{1}+\alpha_{2}-1}\left(z_{1}-z_{2}\right),
$$

- the star-triangle relation

$$
\begin{aligned}
& \frac{1}{\pi} \int \prod_{k=1}^{3} s_{\alpha_{k}}\left(z_{k}-z\right) \mathrm{d}^{2} z \\
& \quad=\left(\prod_{k=1}^{3} \boldsymbol{\Gamma}\left(1-\alpha_{k}\right)\right) \boldsymbol{s}_{1-\alpha_{1}}\left(z_{2}-z_{3}\right) \boldsymbol{s}_{1-\alpha_{2}}\left(z_{3}-z_{1}\right) \boldsymbol{s}_{1-\alpha_{3}}\left(z_{1}-z_{2}\right),
\end{aligned}
$$

which holds provided $\alpha_{1}+\alpha_{2}+\alpha_{3}=\bar{\alpha}_{1}+\bar{\alpha}_{2}+\bar{\alpha}_{3}=2$.

In fact these two relations are equivalent: equation (4.2) is reduced to equation (4.1) in the limit $z_{3} \rightarrow \infty$ and, vice versa, equation (4.2) can be derived from equation (4.1) by a $\operatorname{SL}(2, \mathbb{C})$ transformation. The relation (4.2) underlies the integrability of the noncompact $\mathrm{SL}(2, \mathbb{C})$ spin chain magnets.

In $[5,32,33]$ new solutions of the star-triangle relation have been derived from the elliptic star-triangle relation $[6,18,56,59]$. Below we show that these star-triangle relations can be derived from the integrals (2.3).

First we consider the relation associated with the integral (2.3a). To this end we define the propagator

$$
\begin{aligned}
\boldsymbol{S}_{\alpha}(u) & =(-1)^{[\alpha / 2+u]} \boldsymbol{\Gamma}\left(\frac{1-\alpha}{2}+u\right) \boldsymbol{\Gamma}\left(\frac{1-\alpha}{2}-u\right) \\
& =(-1)^{[\alpha / 2+u]} \frac{\Gamma\left(\frac{1-\alpha}{2}+u\right)}{\Gamma\left(\frac{1+\bar{\alpha}}{2}-\bar{u}\right)} \frac{\Gamma\left(\frac{1-\alpha}{2}-u\right)}{\Gamma\left(\frac{1+\bar{\alpha}}{2}+\bar{u}\right)} .
\end{aligned}
$$

The variables $u$ and $\alpha$ have the form

$$
u=n / 2+\mathrm{i} \nu, \quad \bar{u}=-n / 2+\mathrm{i} \nu, \quad \alpha=m+\sigma, \quad \bar{\alpha}=-m+\sigma,
$$

where $[u]=n$ and $\frac{1}{2}[\alpha]=m$ are either both integer or half-integer numbers and $\langle u\rangle=\nu$, $\langle\alpha\rangle=\sigma$ are real complex numbers, respectively. Under these conditions the arguments of the $\boldsymbol{\Gamma}$-functions in (4.3) have the form (2.1). Slightly abusing the terminology we call the $u(\alpha)$ even or odd depending on the character of $n(m)$ and refer to this property as parity. Also, in order to avoid possible misunderstanding due to our agreement to indicate only the "holomorphic" arguments of functions, $f(\alpha) \equiv f(\alpha, \bar{\alpha})$, we accept that, whenever $\bar{x}$ is not explicitly defined, $x+\alpha \equiv(x+\alpha, x+\bar{\alpha})$, e.g., $\boldsymbol{\Gamma}(1 / 2+z) \equiv \boldsymbol{\Gamma}(1 / 2+z, 1 / 2+\bar{z})$.

The propagator $\boldsymbol{S}_{\alpha}$ inherits many properties of $\boldsymbol{s}_{\alpha}$ :

- for even(odd) $\alpha$ the propagator is an even(odd) function of $u$

$$
\boldsymbol{S}_{\alpha}(-u)=(-1)^{[\alpha]} \boldsymbol{S}_{\alpha}(u),
$$

- for imaginary $\langle\alpha\rangle=\sigma, \boldsymbol{S}_{\alpha}(u)\left(\boldsymbol{S}_{\alpha}(u)\right)^{\dagger}=1$, i.e., the propagator reduces to a phase factor, while for $[\alpha]=0$ and $\langle\alpha\rangle$ real, $\boldsymbol{S}_{\alpha}(u)$ is real and positive.

\footnotetext{
${ }^{2}$ Let us stress here that $[z]^{\alpha}$ denotes the power function while $[\alpha]$ without any superscript stands for the "integer" part of $\alpha,[\alpha]=\alpha-\bar{\alpha}$. We hope that this slightly unfortunate notation does not lead to confusion.
} 
The chain relation for the propagator $\boldsymbol{S}$ follows from the integral (2.3a) for $N=1$. Namely, making the substitution

$$
\begin{array}{ll}
z_{1}=\left(1-\alpha_{1}\right) / 2+z, & w_{1}=\left(1-\alpha_{1}\right) / 2-z, \\
z_{2}=\left(1-\alpha_{2}\right) / 2+w, & w_{2}=\left(1-\alpha_{2}\right) / 2-w
\end{array}
$$

in $(2.3 \mathrm{a})$ one obtains

$$
\sum_{n=-\infty}^{\infty} \int_{-\mathrm{i} \infty}^{\mathrm{i} \infty} \boldsymbol{S}_{\alpha_{1}}(z-u) \boldsymbol{S}_{\alpha_{2}}(u-w) \frac{\mathrm{d} \nu}{2 \pi \mathrm{i}}=\frac{\boldsymbol{\Gamma}\left(1-\alpha_{1}\right) \boldsymbol{\Gamma}\left(1-\alpha_{2}\right)}{\boldsymbol{\Gamma}\left(2-\alpha_{1}-\alpha_{2}\right)} \boldsymbol{S}_{\alpha_{1}+\alpha_{2}-1}(z-w)
$$

where $u=n / 2+\nu$ and sum goes over integers. The parity of the $\alpha_{1}$ and $z$ ( $\alpha_{2}$ and $w$ ) is always the same. The integral (4.4) is well defined provided $\operatorname{Re}\left\langle\alpha_{k}\right\rangle<1, k=1,2$ and $\operatorname{Re}\left\langle\alpha_{1}+\alpha_{2}\right\rangle>1$ : the poles of the $\boldsymbol{\Gamma}$ functions in the integral (4.4) are separated by the integration contour if $\operatorname{Re}\left\langle\alpha_{k}\right\rangle<1$ and the integral converges at large $u$ if $\operatorname{Re}\left\langle\alpha_{1}+\alpha_{2}\right\rangle>1$.

The star-triangle relation for $\boldsymbol{S}_{\alpha}$ can be obtained from the integral identity (3.5) for $N=2$. Let us make the following substitution

$$
z_{i} \mapsto \frac{1-\alpha_{i}}{2}+z_{i}, \quad \bar{z}_{i} \mapsto \frac{1-\bar{\alpha}_{i}}{2}+\bar{z}_{i}, \quad w_{i} \mapsto \frac{1-\alpha_{i}}{2}-z_{i}, \quad \bar{w}_{i} \mapsto \frac{1-\bar{\alpha}_{i}}{2}-\bar{z}_{i}
$$

in that equation. Taking into account that the condition $\sum_{k}\left(z_{k}+w_{k}\right)=1$ gives rise to the following restriction on the indices: $\sum_{k} \alpha_{k}=\sum_{k} \bar{\alpha}_{k}=2$, one derives after some algebra

$$
\begin{aligned}
& \sum_{n=-\infty}^{\infty} \int_{-\mathrm{i} \infty}^{\mathrm{i} \infty} \prod_{k=1}^{3} \boldsymbol{S}_{\alpha_{k}}\left(z_{k}-u\right) \frac{\mathrm{d} \nu}{2 \pi \mathrm{i}} \\
& \quad=\left[\prod_{k=1}^{3} \boldsymbol{\Gamma}\left(1-\alpha_{k}\right)\right] \boldsymbol{S}_{1-\alpha_{1}}\left(z_{2}-z_{3}\right) \boldsymbol{S}_{1-\alpha_{2}}\left(z_{3}-z_{1}\right) \boldsymbol{S}_{1-\alpha_{3}}\left(z_{1}-z_{2}\right) .
\end{aligned}
$$

Here, again, the $\alpha_{k}$ and $z_{k}$ are even or odd simultaneously. For the special choice of the parameters, $\alpha_{k}=\bar{\alpha}_{k}$, this relation coincides with the star-triangle relation [5, equation (22)].

Proceeding with the second integral $(2.3 \mathrm{~b})$ we define the propagator as the product of four $\boldsymbol{\Gamma}$ functions

$$
\mathcal{D}_{\alpha}\left(z_{1}, z_{2}\right)=\boldsymbol{\Gamma}\left(\frac{1-\alpha}{2} \pm z_{1} \pm z_{2}\right)=\frac{\Gamma\left(\frac{1-\alpha}{2} \pm z_{1} \pm z_{2}\right)}{\Gamma\left(\frac{1+\bar{\alpha}}{2} \pm \bar{z}_{1} \pm \bar{z}_{2}\right)}
$$

The requirement for the arguments of $\boldsymbol{\Gamma}$ functions, $\frac{1-\alpha}{2} \pm z_{1} \pm z_{2}$, to be integers imposes obvious restrictions on the relative parity of all variables. Obviously, the propagator $\mathcal{D}_{\alpha}\left(z_{1}, z_{2}\right)$ is an even function of $z_{1}, z_{2}$ and invariant under the $z_{1} \leftrightarrow z_{2}$ permutation. Therefore, for each variable $z_{k}=n_{k} / 2+\nu_{k}$ one can restrict $n_{k}$ to positive (negative) values. Note that unlike the previous case the propagator $\mathcal{D}_{\alpha}$ is not shift invariant. Also, up to a phase factor depending on the parity of arguments, $\mathcal{D}_{\alpha}\left(z_{1}, z_{2}\right) \sim \boldsymbol{S}_{\alpha}\left(z_{1}-z_{2}\right) \boldsymbol{S}_{\alpha}\left(z_{1}+z_{2}\right)$.

The chain relation for the propagator $\mathcal{D}_{\alpha}$ follows from the identity $(2.3 \mathrm{~b})$. Indeed, after the substitution $z_{1(2)}=\left(1-\alpha_{1}\right) / 2 \pm z, z_{3(4)}=\left(1-\alpha_{2}\right) / 2 \pm w$ the integral $(2.3 \mathrm{~b})$ takes the form

$$
2 \int_{ \pm} \mathcal{D} u\|u\|^{2} \mathcal{D}_{\alpha_{1}}(z, u) \mathcal{D}_{\alpha_{2}}(u, w)= \pm \frac{\boldsymbol{\Gamma}\left(1-\alpha_{1}\right) \boldsymbol{\Gamma}\left(1-\alpha_{2}\right)}{\boldsymbol{\Gamma}\left(2-\alpha_{1}-\alpha_{2}\right)} \mathcal{D}_{\alpha_{1}+\alpha_{2}-1}(z, w) .
$$

Here the subscripts \pm indicate that the sum goes over all integers ("+") or half-integers ("-") and we also recall that for $u=n / 2+i \nu,\|u\|^{2}=\nu^{2}+n^{2} / 4$. 
Next, substituting $z_{2 i-1}=\left(1-\alpha_{i}\right) / 2+z_{i}, z_{2 i}=\left(1-\alpha_{i}\right) / 2-z_{i}$, for $i=1,2,3$ in equation $(3.8)$ for $N=2$ one gets

$$
\begin{aligned}
& 2 \int_{ \pm} \mathcal{D} u\|u\|^{2} \prod_{k=1}^{3} \mathcal{D}_{\alpha_{k}}\left(z_{k}, u\right) \\
& \quad=\left(\prod_{k=1}^{3} \Gamma\left(1-\alpha_{k}\right)\right) \mathcal{D}_{1-\alpha_{1}}\left(z_{2}, z_{3}\right) \mathcal{D}_{1-\alpha_{2}}\left(z_{1}, z_{3}\right) \mathcal{D}_{1-\alpha_{3}}\left(z_{1}, z_{2}\right)
\end{aligned}
$$

where $\sum_{k} \alpha_{k}=\sum_{k} \bar{\alpha}_{k}=2$. As was mentioned earlier the parity of all variables have to be coordinated so that (4.6) encompasses four different identities:

(1) All $\alpha_{k}$ are integer / have positive parity

(a) $z_{k}, u$ are even,

(b) $z_{k}, u$ are odd,

(2) $\alpha_{1}$ and $\alpha_{2}, \alpha_{3}$ are even and odd, respectively

(a) $u$ and $z_{1}$ are even and $z_{2}, z_{3}$ are odd,

(b) $u$ and $z_{1}$ are odd and $z_{2}, z_{3}$ are even.

Variant (1a) corresponds to the star-triangle relation obtained by Kels [32, equation (17)]. An extension of the star-triangle relation of [32] which follows from the relation (3.8) with $N=2$ was also considered by Sarkissian and Spiridonov [55].

In Cases (1a) and (1b) the functions $\mathcal{D}_{\alpha}\left(z_{1}, z_{2}\right)$ are real if $0<\alpha=\bar{\alpha}<1: \mathcal{D}_{\alpha}\left(z_{1}, z_{2}\right)>0$ for even $z_{1}, z_{2}$ (Case (1a)) and $-\mathcal{D}_{\alpha}\left(z_{1}, z_{2}\right)>0$ for odd $z_{1}, z_{2}$ (Case (1b)). In both cases these functions can be interpreted as the Boltzmann weights of lattice integrable models, for more details see references $[32,33]$.

\section{Quasi-classical limit}

Let us consider the identities (4.4), (4.5) when the external variables become large. We replace the variables $z_{k}\left(w_{k}\right)$ in (4.4), (4.5) by $L z_{k}=L\left(x_{k}+\mathrm{i} y_{k}\right)$ and take the limit $L \rightarrow \infty$. The variables $x_{k}, y_{k}$ can be considered in this limit as continuous ones, so that $z_{k} \in \mathbb{C}, \bar{z}_{k}=z_{k}^{*}$. It is easy to check that the leading contribution to the integrals (4.4), (4.5) comes from the region where $u_{k} \sim L$, so that we replace $u_{k} \mapsto L u_{k}$ as well. Moreover in this limit $\boldsymbol{S}_{\alpha}$ turns into $\boldsymbol{s}_{\alpha}$ :

$$
L^{2\langle\alpha\rangle} \boldsymbol{S}_{\alpha}(L z) \underset{L \rightarrow \infty}{\mapsto} z^{-\alpha} \bar{z}^{-\bar{\alpha}}=\boldsymbol{s}_{\alpha}(z)
$$

The sum over $n$ in $\int \mathcal{D} u$ can be replaced by the integral so that

$$
\frac{1}{L^{2}} \sum_{n=-\infty}^{\infty} \int_{-\mathrm{i} \infty}^{\mathrm{i} \infty} \frac{\mathrm{d} \nu}{2 \pi \mathrm{i}} \underset{L \rightarrow \infty}{\mapsto} \frac{1}{\pi} \int \mathrm{d}^{2} u,
$$

where $u=u_{x}+\mathrm{i} u_{y}$ and $\mathrm{d}^{2} z=\mathrm{d} u_{x} \mathrm{~d} u_{y}$. Taking into account equations (5.1) and (5.2) one checks that in the limit $L \rightarrow \infty$ equations (4.4), (4.5) become the chain and the star-triangle relations, equations (4.1) and (4.2), respectively.

In what follows we study the quasi-classical limit the integrals (2.3a) and (3.5) for general $N$. First of all we rewrite these identities in term of the propagator $\boldsymbol{S}_{\alpha}$. After the change of variables

$$
z_{k} \mapsto \frac{1-\alpha_{k}}{2}+z_{k}, \quad w_{k} \mapsto \frac{1-\alpha_{k}}{2}-z_{k}, \quad k=1, \ldots, N+1 .
$$


Equation (2.3a) takes the following form

$$
\begin{aligned}
\frac{1}{N !} \int \mathcal{D} u_{1} \cdots \mathcal{D} u_{N} \prod_{1 \leq i<j \leq N}\left\|u_{i}-u_{j}\right\|^{2} \prod_{k=1}^{N} \prod_{m=1}^{N+1} \boldsymbol{S}_{\alpha_{m}}\left(z_{m}-u_{k}\right) \\
=(-1)^{\sum_{m=1}^{N} m\left[\alpha_{m+1}\right]} \frac{\prod_{k=1}^{N+1} \boldsymbol{\Gamma}\left(1-\alpha_{k}\right)}{\boldsymbol{\Gamma}\left(N+1-\sum_{k=1}^{N+1} \alpha_{k}\right)} \prod_{1 \leq i<k \leq N+1} \boldsymbol{S}_{\alpha_{i}+\alpha_{k}-1}\left(z_{i}-z_{k}\right) .
\end{aligned}
$$

Similarly, the identity (3.5) can be represented as follows

$$
\begin{gathered}
\frac{1}{(N-1) !} \int \mathcal{D} u_{1} \cdots \mathcal{D} u_{N-1} \prod_{1 \leq i<j \leq N-1}\left\|u_{i}-u_{j}\right\|^{2} \prod_{k=1}^{N} \prod_{m=1}^{N+1} \boldsymbol{S}_{\alpha_{m}}\left(z_{m}-u_{k}\right) \\
=(-1)^{\sum_{m=1}^{N} m\left[\alpha_{m+1}\right]}\left(\prod_{k=1}^{N+1} \boldsymbol{\Gamma}\left(1-\alpha_{k}\right)\right) \prod_{1 \leq i<k \leq N+1} \boldsymbol{S}_{\alpha_{i}+\alpha_{k}-1}\left(z_{i}-z_{k}\right)
\end{gathered}
$$

and where $\sum_{k} \alpha_{k}=\sum_{k} \bar{\alpha}_{k}=N$.

In the quasi-classical limit these identities are reduced to the following two-dimensional integrals with power functions

$$
\begin{gathered}
\frac{1}{N !} \prod_{1 \leq i<j \leq N}\left|u_{i}-u_{j}\right|^{2} \prod_{k=1}^{N} \prod_{m=1}^{N+1}\left[z_{m}-u_{k}\right]^{-\alpha_{m}} \mathrm{~d}^{2} u_{1} \cdots \mathrm{d}^{2} u_{N} \\
=\pi^{N}(-1)^{\sum_{m=1}^{N} m\left[\alpha_{m+1}\right]} \frac{\prod_{k=1}^{N+1} \Gamma\left(1-\alpha_{k}\right)}{\Gamma\left(N+1-\sum_{k=1}^{N+1} \alpha_{k}\right)} \prod_{1 \leq i<k \leq N+1}\left[z_{i}-z_{k}\right]^{1-\alpha_{i}-\alpha_{k}}
\end{gathered}
$$

and

$$
\begin{aligned}
& \frac{1}{(N-1) !} \int \prod_{1 \leq i<j \leq N-1}\left|u_{i}-u_{j}\right|^{2} \prod_{k=1}^{N} \prod_{m=1}^{N+1}\left[z_{m}-u_{k}\right]^{-\alpha_{m}} \mathrm{~d}^{2} u_{1} \cdots \mathrm{d}^{2} u_{N-1} \\
& \quad=\pi^{N-1}(-1)^{\sum_{m=1}^{N} m\left[\alpha_{m+1}\right]}\left(\prod_{k=1}^{N+1} \Gamma\left(1-\alpha_{k}\right)\right) \prod_{1 \leq i<k \leq N+1}\left[z_{i}-z_{k}\right]^{1-\alpha_{1}-\alpha_{k}}
\end{aligned}
$$

It can be checked that in the quasi-classical limit the propagator $\mathcal{D}_{\alpha}(z, w)$ turns into $\left[z^{2}-w^{2}\right]^{-\alpha}$. Therefore equations (2.3b) and equation (3.7) do not produce new identities in this limit but reduce to the integrals (5.3) and (5.4) after an appropriate change of variables.

One can take a different point of view on the integrals (2.3a) and (2.3b) and consider them as the "quantized" version of the integrals (5.3). For $\alpha_{k}=\bar{\alpha}_{k}$ the integral (5.3) is the special case of the duality relation [2] for the Dotsenko-Fateev (DF) integrals [19] considered in the next section. Therefore one can hope that there exists a "quantized" version of the duality relation in a general situation. 


\section{Dotsenko-Fateev integrals}

In this section we give an elementary proof of the following relation

$$
\begin{aligned}
& \frac{1}{\pi^{n} n !} \int \prod_{i<k}^{n}\left[y_{i}-y_{k}\right] \prod_{i=1}^{n} \prod_{j=1}^{n+m+1}\left[y_{i}-z_{j}\right]^{-\alpha_{j}} \mathrm{~d}^{2} y_{1} \cdots \mathrm{d}^{2} y_{n}=\frac{(-1)^{\sum_{k=1}^{n+m} k\left[\alpha_{k+1}\right]} \prod_{j=1}^{n+m+1} \boldsymbol{\Gamma}\left(1-\alpha_{j}\right)}{\boldsymbol{\Gamma}\left(1+n-\sum_{j=1}^{n+m+1} \alpha_{j}\right)} \\
& \times \prod_{i<j}^{n+m+1}\left[z_{j}-z_{i}\right]^{1-\alpha_{i}-\alpha_{j}} \frac{1}{\pi^{m} m !} \int \prod_{i<k}^{m}\left[u_{i}-u_{k}\right] \prod_{i=1}^{m} \prod_{j=1}^{n+m+1}\left[u_{i}-z_{j}\right]^{-1+\alpha_{j}} \mathrm{~d}^{2} u_{1} \cdots \mathrm{d}^{2} u_{m}, \quad
\end{aligned}
$$

which is, provided the parameters satisfy the constraint $\alpha_{k}=\bar{\alpha}_{k}$, the duality relation [2,21] for the DF integrals [19], see also [46]. For $m=0$ this identity coincides with (5.3).

To evaluate the integral on the l.h.s. of (6.1) we go over to the variables $x_{k}=x_{k}\left(y_{1}, \ldots, y_{n}\right)$ which are essentially the elementary symmetric functions

$$
\prod_{i=1}^{n}\left(y_{i}+t\right)=x_{1}+x_{2} t+x_{3} t^{2}+\cdots+x_{n} t^{n-1}+t^{n}
$$

Every point in $x$-space has $n$ ! preimages in $y$-space and the Jacobian of the transformation is $J=\left|\frac{\partial x_{k}}{\partial y_{i}}\right|^{2}=\prod_{i<j}^{n}\left[y_{i}-y_{k}\right]$. Introducing the notation $t_{j}=-z_{j}$ one gets for the integral on the 1.h.s. of $(6.1)$

$$
\pi^{-n} \int_{j=1}^{n+m+1}\left[x_{1}+x_{2} t_{j}+x_{3} t_{j}^{2}+\cdots+x_{n} t_{j}^{n-1}+t_{j}^{n}\right]^{-\alpha_{j}} \mathrm{~d}^{2} x_{1} \cdots \mathrm{d}^{2} x_{n} .
$$

Then using the momentum space representation for the propagators

$$
[z]^{-\alpha}=\frac{1}{\pi} \mathrm{i}^{\alpha-\bar{\alpha}} \boldsymbol{\Gamma}(1-\alpha) \int \mathrm{e}^{\mathrm{i}(p z+\bar{z} \bar{p})}[p]^{\alpha-1} \mathrm{~d}^{2} p
$$

in (6.2) and carrying out all integrals over $x_{k}$ one gets

$$
\begin{aligned}
& \frac{\mathrm{i}^{\sum_{j}\left(\alpha_{j}-\bar{\alpha}_{j}\right)}}{\pi^{m+1}} \prod_{k=1}^{n+m+1} \boldsymbol{\Gamma}\left(1-\alpha_{k}\right) \prod_{i=1}^{n+m+1} \int \mathrm{d}^{2} p_{i}\left[p_{i}\right]^{\alpha_{k}-1} \prod_{k=1}^{n} \delta^{(2)}\left(\sum_{j} p_{j} t_{j}^{k-1}\right) \mathrm{e}^{\mathrm{i} \sum_{j}\left(p_{j} t_{j}^{n}+\bar{p}_{j} \bar{t}_{j}^{n}\right)} \\
& =\frac{\prod_{k=1}^{n+m+1} \boldsymbol{\Gamma}\left(1-\alpha_{k}\right)}{\pi^{m} \boldsymbol{\Gamma}\left(n+1-\sum_{j} \alpha_{j}\right)} \prod_{i=1}^{n+m+1} \int \frac{\mathrm{d}^{2} p_{i}}{\left[p_{i}\right]^{1-\alpha_{i}}} \prod_{k=1}^{n} \delta^{(2)}\left(\sum_{j} p_{j} t_{j}^{k-1}\right) \delta^{(2)}\left(\sum_{j} p_{j} t_{j}^{n}-1\right) .
\end{aligned}
$$

In order to get the last $\delta$-function one represents $\mathrm{e}^{\mathrm{i} \sum_{j}\left(p_{j} t_{j}^{n}+\bar{p}_{j} \bar{t}_{j}^{n}\right)}$ as $\int \mathrm{e}^{\mathrm{i}(\lambda+\bar{\lambda})} \delta^{(2)}\left(\lambda-\sum_{j} p_{j} t_{j}^{n}\right) d \lambda$ and rescales $p_{j} \rightarrow \lambda p_{j}$. The delta functions cut out a $m$-dimensional subspace in the $(n+m+1)$ dimensional space defined by the linear equations

$$
\sum_{j=1}^{n+m+1} p_{j} t_{j}^{k-1}=0, \quad k=1, \ldots, n \quad \text { and } \quad \sum_{j=1}^{n+m+1} p_{j} t_{j}^{n}=1 .
$$

The solution depends on $m$ free variables, $u_{1}, \ldots, u_{m}$, and takes the form

$$
p_{j}\left(u_{1}, \ldots, u_{m}\right)=\lambda_{j}\left(u_{1}+t_{j}\right) \cdots\left(u_{m}+t_{j}\right),
$$


where $\lambda_{j}=\prod_{k \neq j}\left(t_{j}-t_{k}\right)^{-1}, j=1, \ldots, n+m+1$. That $p_{j}$, (6.4), satisfy equations (6.3) follows from an identity due to Milne [41, Lemma 1.33], see also [48, equation (2.3)],

$$
\sum_{k=1}^{N} \frac{\left(b_{1}-t_{k}\right) \cdots\left(b_{N}-t_{k}\right)}{t_{k} \prod_{j \neq k}\left(t_{j}-t_{k}\right)}=\frac{b_{1} \cdots b_{N}}{t_{1} \cdots t_{N}}-1 .
$$

To see it is enough to put $N=m+n+1, b_{k}=-u_{k}$ for $k \leq m$ and $b_{k}=-u$, for $k>m$ in the above identity and compare the coefficients at the powers $u^{k}, k=0, \ldots, n$ on both sides of (6.5).

Making the change of variables

$$
\begin{array}{ll}
p_{j}=\lambda_{j}\left(u_{1}+t_{j}\right) \cdots\left(u_{m}+t_{j}\right)+s_{j}, & j=1, \ldots, n+1, \\
p_{j}=\lambda_{j}\left(u_{1}+t_{j}\right) \cdots\left(u_{m}+t_{j}\right), & j=n+2, \ldots, n+m+1,
\end{array}
$$

and taking into account that

$$
\prod_{k=1}^{n} \delta^{2}\left(\sum_{j=1}^{n+m+1} p_{j} t_{j}^{k-1}\right) \delta^{2}\left(\sum_{j=1}^{n+m+1} p_{j} t_{j}^{n}-1\right)=\prod_{k=1}^{n+1} \delta^{2}\left(\sum_{j=1}^{n+1} s_{j} t_{j}^{k-1}\right)=\frac{\prod_{j=1}^{n+1} \delta^{2}\left(s_{j}\right)}{\prod_{1 \leq i<j \leq n+1}\left[t_{i}-t_{j}\right]}
$$

and

$$
\prod_{j=1}^{n+m+1} \mathrm{~d}^{2} p_{j}=\left(\prod_{n+2 \leq i<j \leq n+m+1}\left[t_{i}-t_{j}\right] \prod_{k=n+2}^{n+m+1}\left[\lambda_{k}\right]\right) \frac{1}{m !} \prod_{j=1}^{m} \mathrm{~d}^{2} u_{j} \prod_{j=1}^{n+1} \mathrm{~d}^{2} s_{j}
$$

one gets after some algebra

$$
\begin{aligned}
& \frac{\prod_{k=1}^{n+m+1} \boldsymbol{\Gamma}\left(1-\alpha_{k}\right)}{\boldsymbol{\Gamma}\left(n+1-\sum_{j} \alpha_{j}\right)}\left(\prod_{j<k}(-1)^{\alpha_{k}-\bar{\alpha}_{k}}\left[t_{j}-t_{k}\right]^{1-\alpha_{k}-\alpha_{j}}\right) \\
& \quad \times \frac{1}{\pi^{m} m !} \prod_{j=1}^{n+m+1} \int \prod_{k=1}^{m}\left(u_{k}+t_{j}\right)^{\alpha_{j}-1} \mathrm{~d}^{2} u_{1} \cdots \mathrm{d}^{2} u_{m} .
\end{aligned}
$$

It coincides with the r.h.s. of equation (6.1) after changing $t_{j} \rightarrow-z_{j}$. We have learned from the discussions with Litvinov that similar proof of the duality relation (6.1) is presented in his lectures on conformal field theory [39].

Finally, we make a conjecture that the duality relation (6.1) admits a generalization to the "quantized" cases. Namely, these relations take the form

$$
\begin{aligned}
\frac{1}{n !} \int \mathcal{D} u_{1} \cdots \mathcal{D} u_{n} \prod_{i<j}\left\|u_{i}-u_{j}\right\|^{2} \prod_{i=1}^{n} \prod_{j=1}^{n+m+1}(-1)^{\left[u_{i}\right]} \boldsymbol{\Gamma}\left(z_{j}-u_{i}\right) \boldsymbol{\Gamma}\left(u_{i}+w_{j}\right) \\
=\frac{\prod_{i, j=1}^{n+m+1} \boldsymbol{\Gamma}\left(z_{i}+w_{j}\right)}{\boldsymbol{\Gamma}\left(\sum_{j}\left(z_{j}+w_{j}\right)-m\right)}(-1)^{m \sum_{j}\left[z_{j}-w_{j}\right]} \frac{1}{m !} \int \mathcal{D} u_{1} \cdots \mathcal{D} u_{m} \\
\quad \times \prod_{i<j}\left\|u_{i}-u_{j}\right\|^{2} \prod_{i=1}^{m} \prod_{j=1}^{n+m+1}(-1)^{\left[u_{i}\right]} \boldsymbol{\Gamma}\left(z_{j}^{\prime}-u_{i}\right) \boldsymbol{\Gamma}\left(w_{j}^{\prime}+u_{i}\right)
\end{aligned}
$$


where $z_{j}^{\prime}=1 / 2-w_{j}, w_{j}^{\prime}=1 / 2-z_{j}$ and

$$
\begin{gathered}
\frac{2^{n}}{n !} \int_{ \pm} \mathcal{D} u_{1} \cdots \mathcal{D} u_{n}\left\|u_{k}\right\|^{2} \prod_{1 \leq i<j \leq n}\left\|u_{i} \pm u_{j}\right\|^{2} \prod_{i=1}^{n} \prod_{j=1}^{2(n+m+1)} \Gamma\left(z_{j} \pm u_{i}\right) \\
=\varkappa_{n+m} \frac{\prod_{i<j}^{2(n+m+1)} \Gamma\left(z_{i}+z_{j}\right)}{\Gamma\left(\sum_{j} z_{j}-m\right)} \frac{2^{m}}{m !} \int_{ \pm} \mathcal{D} u_{1} \cdots \mathcal{D} u_{m}\left\|u_{k}\right\|^{2} \\
\quad \times \prod_{1 \leq i<j \leq m}\left\|u_{i} \pm u_{j}\right\|^{2} \prod_{i=1}^{m} \prod_{j=1}^{2(n+m+1)} \boldsymbol{\Gamma}\left(1 / 2-z_{j} \pm u_{i}\right),
\end{gathered}
$$

where $\varkappa_{k}=1,(-1)^{k(k+1) / 2}$ for the integer and half-integer cases, respectively. For $m=0$ these integrals are equivalent to the integrals (2.3) and in the quasi-classical limit both of them reproduce the duality relation (6.1). For $n=m=1$ the relations (6.6) and (6.7) follow from the star-triangle relations (4.5) and (4.6). For few first $n$ and $m$ the integrals (6.6), (6.7) go through numerical tests. Closing this section we note that quite similar duality relations were observed recently in the so-called conformal fishnet model [11].

\section{Summary}

In $[16,17]$ a generalization of Gustafson integrals to the complex case have been obtained. The derivation of these integrals rely on the completeness of the SoV representation for the $\operatorname{SL}(2, \mathbb{C})$ magnets, that is not yet proven. In this work we presented a direct calculation of two $\boldsymbol{\Gamma}$ function integrals. We expect that these integral identities will be helpful in proving the completeness of the $\mathrm{SoV}$ representation for the $\mathrm{SL}(2, \mathbb{C})$ spin chains.

The complex integrals are, up to appropriate modification of the $\Gamma$ function and integration measure, exact copies of the integrals obtained by R.A. Gustafson [28]. However, the analytic properties of these integrals are different. We have shown that several, apparently distinct in the $\mathrm{SL}(2, \mathbb{R})$ context integrals are intrinsically related to each other in the $\mathrm{SL}(2, \mathbb{C})$ formulation.

We have also shown that the complex $\boldsymbol{\Gamma}$ integrals for the lowest $N$ underlie the star-triangle identities derived in references $[5,32,33]$ and in the quasi-classical limit are reduced to the special $(m=0)$ case of the duality relation for the DF integrals [2, 19, 21]. We also conjecture an appropriate modification of these duality relations to hold for the integrals with complex $\boldsymbol{\Gamma}$ functions.

\section{Acknowledgements}

We are grateful to A.V. Litvinov and V.P. Spiridonov for fruitful discussions. We would also like to express our sincere gratitude to the referees for their numerous useful comments and suggestions. This study was supported by the Russian Science Foundation project No 19-1100131 and Deutsche Forschungsgemeinschaft (A.M.), grant MO 1801/1-3.

\section{References}

[1] Andrews G.E., Askey R., Roy R., Special functions, Encyclopedia of Mathematics and its Applications, Vol. 71, Cambridge University Press, Cambridge, 1999.

[2] Baseilhac P., Fateev V.A., Expectation values of local fields for a two-parameter family of integrable models and related perturbed conformal field theories, Nuclear Phys. B 532 (1998), 567-587, arXiv:hep-th/9906010.

[3] Baxter R.J., Exactly solved models in statistical mechanics, Academic Press, Inc., London, 1982. 
[4] Bazhanov V.V., Kels A.P., Sergeev S.M., Quasi-classical expansion of the star-triangle relation and integrable systems on quad-graphs, J. Phys. A: Math. Theor. 49 (2016), 464001, 44 pages, arXiv:1602.07076.

[5] Bazhanov V.V., Mangazeev V.V., Sergeev S.M., Exact solution of the Faddeev-Volkov model, Phys. Lett. A 372 (2008), 1547-1550, arXiv:0706.3077.

[6] Bazhanov V.V., Sergeev S.M., A master solution of the quantum Yang-Baxter equation and classical discrete integrable equations, Adv. Theor. Math. Phys. 16 (2012), 65-95, arXiv:1006.0651.

[7] Beisert N., Ahn C., Alday L.F. et al., Review of AdS/CFT integrability: an overview, Lett. Math. Phys. 99 (2012), 3-32, arXiv:1012.3982.

[8] Belitsky A.V., Braun V.M., Gorsky A.S., Korchemsky G.P., Integrability in QCD and beyond, Internat. J. Modern Phys. A 19 (2004), 4715-4788, arXiv:hep-th/0407232.

[9] Belitsky A.V., Derkachov S.E., Manashov A.N., Quantum mechanics of null polygonal Wilson loops, Nuclear Phys. B 882 (2014), 303-351, arXiv:1401.7307.

[10] Cavaglià A., Gromov N., Levkovich-Maslyuk F., Separation of variables and scalar products at any rank, J. High Energy Phys. 2019 (2019), no. 0, 052, 28 pages, arXiv:1907.03788.

[11] Derkachov S., Kazakov V., Olivucci E., Basso-Dixon correlators in two-dimensional fishnet CFT, J. High Energy Phys. 2019 (2019), no. 4, 032, 31 pages, arXiv:1811.10623.

[12] Derkachov S.E., Korchemsky G.P., Manashov A.N., Noncompact Heisenberg spin magnets from highenergy QCD. I. Baxter Q-operator and separation of variables, Nuclear Phys. B 617 (2001), 375-440, arXiv:hep-th/0107193.

[13] Derkachov S.E., Kozlowski K.K., Manashov A.N., On the separation of variables for the modular XXZ magnet and the lattice sinh-Gordon models, Ann. Henri Poincaré 20 (2019), 2623-2670, arXiv:1806.04487.

[14] Derkachov S.E., Manashov A.N., Iterative construction of eigenfunctions of the monodromy matrix for SL(2, C) magnet, J. Phys. A: Math. Theor. 47 (2014), 305204, 25 pages, arXiv:1401.7477.

[15] Derkachov S.E., Manashov A.N., Spin chains and Gustafson's integrals, J. Phys. A: Math. Theor. 50 (2017), 294006, 20 pages, arXiv:1611.09593.

[16] Derkachov S.E., Manashov A.N., Valinevich P.A., Gustafson integrals for SL(2, C) spin magnet, J. Phys. A: Math. Theor. 50 (2017), 294007, 12 pages, arXiv:1612.00727.

[17] Derkachov S.E., Manashov A.N., Valinevich P.A., SL(2, C) Gustafson integrals, SIGMA 14 (2018), 030, 16 pages, arXiv:1711.07822.

[18] Derkachov S.E., Spiridonov V.P., Yang-Baxter equation, parameter permutations, and the elliptic beta integral, Russian Math. Surveys 68 (2013), 1027-1072, arXiv:1205.3520.

[19] Dotsenko V.S., Fateev V.A., Four-point correlation functions and the operator algebra in 2D conformal invariant theories with central charge $C \leq 1$, Nuclear Phys. B 251 (1985), 691-734.

[20] Faddeev L.D., How the algebraic Bethe ansatz works for integrable models, in Symétries quantiques (Les Houches, 1995), North-Holland, Amsterdam, 1998, 149-219, arXiv:hep-th/9605187.

[21] Fateev V.A., Litvinov A.V., Multipoint correlation functions in Liouville field theory and minimal Liouville gravity, Theoret. and Math. Phys. 154 (2008), 454-472, arXiv:0707.1664.

[22] Forrester P.J., Warnaar S.O., The importance of the Selberg integral, Bull. Amer. Math. Soc. (N.S.) 45 (2008), 489-534, arXiv:0710.3981.

[23] Gel'fand I.M., Graev M.I., Retakh V.S., Hypergeometric functions over an arbitrary field, Russian Math. Surveys 59 (2004), 831-905.

[24] Gromov N., Levkovich-Maslyuk F., New compact construction of eigenstates for supersymmetric spin chains, J. High Energy Phys. 2018 (2018), no. 9, 085, 23 pages, arXiv:1805.03927.

[25] Gromov N., Levkovich-Maslyuk F., Sizov G., New construction of eigenstates and separation of variables for SU $(N)$ quantum spin chains, J. High Energy Phys. 2017 (2017), no. 9, 111, 40 pages, arXiv:1610.08032.

[26] Gustafson R.A., The Macdonald identities for affine root systems of classical type and hypergeometric series very-well-poised on semisimple Lie algebras, in Ramanujan International Symposium on Analysis (Pune, 1987), Macmillan of India, New Delhi, 1989, 185-224.

[27] Gustafson R.A., Some $q$-beta and Mellin-Barnes integrals with many parameters associated to the classical groups, SIAM J. Math. Anal. 23 (1992), 525-551.

[28] Gustafson R.A., Some $q$-beta and Mellin-Barnes integrals on compact Lie groups and Lie algebras, Trans. Amer. Math. Soc. 341 (1994), 69-119. 
[29] Gustafson R.A., Some $q$-beta integrals on $\mathrm{SU}(n)$ and $\mathrm{Sp}(n)$ that generalize the Askey-Wilson and NasrallahRahman integrals, SIAM J. Math. Anal. 25 (1994), 441-449.

[30] Ismagilov R.S., On Racah operators, Funct. Anal. Appl. 40 (2006), 222-224.

[31] Ismagilov R.S., On Racah operators for representations of the principal series of the group $\operatorname{SL}(2, \mathbb{C}), S b$. Math. 198 (2007), 77-90.

[32] Kels A.P., A new solution of the star-triangle relation, J. Phys. A: Math. Theor. 47 (2014), 055203, 7 pages, arXiv:1302.3025.

[33] Kels A.P., New solutions of the star-triangle relation with discrete and continuous spin variables, J. Phys. A: Math. Theor. 48 (2015), 435201, 19 pages, arXiv:1504.07074.

[34] Kitanine N., Maillet J.M., Niccoli G., Terras V., The open XXX spin chain in the SoV framework: scalar product of separate states, J. Phys. A: Math. Theor. 50 (2017), 224001, 35 pages, arXiv:1606.06917.

[35] Kitanine N., Maillet J.M., Niccoli G., Terras V., The open XXZ spin chain in the SoV framework: scalar product of separate states, J. Phys. A: Math. Theor. 51 (2018), 485201, 46 pages, arXiv:1807.05197.

[36] Kozlowski K.K., Unitarity of the SoV transform for the Toda chain, Comm. Math. Phys. 334 (2015), 223-273, arXiv:1306.4967.

[37] Kulish P.P., Reshetikhin N.Y., Sklyanin E.K., Yang-Baxter equations and representation theory. I, Lett. Math. Phys. 5 (1981), 393-403.

[38] Kulish P.P., Sklyanin E.K., Quantum spectral transform method. Recent developments, in Integrable Quantum Field Theories (Tvärminne, 1981), Lecture Notes in Phys., Vol. 151, Springer, Berlin - New York, 1982, 61-119.

[39] Litvinov A.V., Lectures on conformal field theory, Lecture 6, available at http://www.mathnet.ru/rus/ person27650.

[40] Maillet J.M., Niccoli G., On quantum separation of variables, J. Math. Phys. 59 (2018), 091417, 47 pages, arXiv:1807.11572.

[41] Milne S.C., A $q$-analog of hypergeometric series well-poised in $\mathrm{SU}(n)$ and invariant $G$-functions, Adv. Math. 58 (1985), 1-60.

[42] Milne S.C., A q-analog of the Gauss summation theorem for hypergeometric series in $\mathrm{U}(n)$, Adv. Math. 72 (1988), 59-131.

[43] Molchanov V.F., Neretin Yu.A., A pair of commuting hypergeometric operators on the complex plane and bispectrality, arXiv:1812.06766.

[44] Neretin Yu.A., An analog of the Dougall formula and of the de Branges-Wilson integral, arXiv:1812.07341.

[45] Neretin Yu.A., Barnes-Ismagilov integrals and hypergeometric functions of the complex field, arXiv:1910.10686.

[46] Pasquetti S., Sacchi M., 3d dualities from 2d free field correlators: recombination and rank stabilization, arXiv:1905.05807.

[47] Rains E.M., Spiridonov V.P., Determinants of elliptic hypergeometric integrals, Funct. Anal. Appl. 43 (2009), 297-311, arXiv:0712.4253.

[48] Rosengren H., Elliptic hypergeometric series on root systems, Adv. Math. 181 (2004), 417-447, arXiv:math.CA/0207046.

[49] Ryan P., Volin D., Separated variables and wave functions for rational $\mathfrak{g l}(N)$ spin chains in the companion twist frame, J. Math. Phys. 60 (2019), 032701, 23 pages, arXiv:1810.10996.

[50] Schlosser M.J., Hypergeometric and basic hypergeometric series and integrals associated with root systems, available at https://www.mat.univie.ac.at/ schlosse/AB-mbhs.html.

[51] Schrader G., Shapiro A., On b-Whittaker functions, arXiv:1806.00747.

[52] Sklyanin E.K., Boundary conditions for integrable quantum systems, J. Phys. A: Math. Gen. 21 (1988), $2375-2389$.

[53] Sklyanin E.K., Quantum inverse scattering method. Selected topics, in Quantum Group and Quantum Integrable Systems, Nankai Lectures Math. Phys., World Sci. Publ., River Edge, NJ, 1992, 63-97, arXiv:hep-th/9211111.

[54] Sklyanin E.K., Takhtadzhyan L.A., Faddeev L.D., Quantum inverse problem method. I, Theoret. and Math. Phys. 40 (1979), 688-706. 
[55] Spiridonov V.P., The endless beta integrals, Talk at the Workshop "Elliptic Integrable Systems, Special Functions and Quantum Field Theory" (June 16-20, 2019, Nordita, Stockholm, Sweden).

[56] Spiridonov V.P., On the elliptic beta function, Russian Math. Surveys 56 (2001), 185-186.

[57] Spiridonov V.P., Theta hypergeometric integrals, St. Petersburg Math. J. 15 (2003), 929-967, arXiv:math.CA/0303205.

[58] Spiridonov V.P., Short proofs of the elliptic beta integrals, Ramanujan J. 13 (2007), 265-283, arXiv:math.CA/0408369.

[59] Spiridonov V.P., Elliptic beta integrals and solvable models of statistical mechanics, in Algebraic aspects of Darboux transformations, quantum integrable systems and supersymmetric quantum mechanics, Contemp. Math., Vol. 563, Amer. Math. Soc., Providence, RI, 2012, 181-211, arXiv:1011.3798.

[60] Spiridonov V.P., Vartanov G.S., Elliptic hypergeometry of supersymmetric dualities, Comm. Math. Phys. 304 (2011), 797-874, arXiv:0910.5944.

[61] Spiridonov V.P., Warnaar S.O., Inversions of integral operators and elliptic beta integrals on root systems, Adv. Math. 207 (2006), 91-132, arXiv:math.CA/0411044.

[62] Stokman J.V., On BC type basic hypergeometric orthogonal polynomials, Trans. Amer. Math. Soc. 352 (2000), 1527-1579, arXiv:q-alg/9707005. 